\title{
Apoyo a los SUBSISTEMAS DE TALENTO HUMANO, SELECCIÓN Y RECLUTAMIENTO A PARTIR DE UN SISTEMA EXPERTO. CASO DE ESTUDIO
}

\author{
SUPPORT TO THE HUMAN TALENT \\ SUBSYSTEMS, SELECTION AND RECRUITMENT \\ FROM AN EXPERT SYSTEM. CASE STUDY
}

\author{
Karina Matute-Pinos ${ }^{1, *}$ (D), Rodolfo Bojorque-Chasi ${ }^{2}$
}

Recibido: 10-11-2020, Recibido tras revisión: 04-02-2021, Aceptado: 25-02-2021, Publicado: 01-07-2021

\section{Resumen}

La gestión de talento humano es un factor fundamental en el éxito de las organizaciones. La inclusión en el entorno laboral de las personas con discapacidad ha ayudado a potenciar sus cualidades y a aprovechar su talento. Muchos de los sistemas de gestión de talento humano carecen de directrices para el reclutamiento y selección de una persona con discapacidad, es por eso por lo que el presente trabajo muestra el estudio realizado a estos dos procesos indicando los factores que influyen en la asignación o no de un cargo, donde de cada candidato es considerando el nivel y tipo de discapacidad, nivel de estudios, experiencia, capacitación entre otros aspectos, enfocándose en la tarea de aplicar técnicas de aprendizaje supervisado que permitan clasificar a un candidato con discapacidad para un puesto de trabajo como apto o no y técnicas de aprendizaje no supervisado como el clustering que ayuda a definir patrones ocultos en los datos si los hubiera. El resultado obtenido del estudio presenta varias técnicas de clasificación y la selección de la más adecuada para el conjunto de datos en cuestión, igualmente mediante técnicas de aprendizaje no supervisado se determina cuántos clústeres representativos se identifican en los datos. No se busca que se integren las personas a través de las discapacidades, sino todo lo contrario, que se integren las personas por medio de la potencialidad de todas sus capacidades.

Palabras clave: aprendizaje automático, inteligencia artificial, minería de datos, sistemas expertos

\section{Abstract}

Human talent management is a key factor in the success of organizations. The inclusion of people with disabilities in the work environment has helped to enhance their qualities and harness their talent. Many of the human talent management systems lack guidelines for the recruitment and selection of a person with a disability, this is why this work shows the study carried out on these two processes indicating the factors that influence the allocation or not of a position, where for each candidate it is considered the level and type of disability, level of education, experience, training among other aspects, focusing on the task of applying supervised learning techniques that enable us to classify a candidate with a disability as suitable or not for a job, and unsupervised learning techniques such as clustering that helps us define hidden patterns in the data, if any. The result obtained from the study presents some classifications techniques and the selection of the most appropriate one for the available dataset. It is not sought to integrate people through their disabilities, but quite the opposite, to integrate people based on the potential of all their abilities.

Keywords: automatic learning, artificial intelligence, data mining, expert systems

\footnotetext{
${ }_{1, *}$ Maestría en Administración de Empresas, Universidad Politécnica Salesiana, Ecuador.

Autor para correspondencia kmatute@est.ups.educ.ec.

${ }^{2}$ Universidad Politécnica Salesiana, Ecuador.
}

Forma sugerida de citación: Matute-Pinos, K. y Bojorque-Chasi, R. (2021). «Apoyo a los subsistemas de talento humano, selección y reclutamiento a partir de un sistema experto. Caso de estudio». InGENiUs. N. ${ }^{\circ} 26$, (julio-diciembre). pp. 41-48. DOI: https://doi.org/10.17163/ings.n26.2021.04. 


\section{Introducción}

En los últimos años distintos organismos y Gobiernos han considerado como punto importante en su gestión el mejorar la calidad de vida de las personas con discapacidad, tal es el ejemplo del Gobierno de Ecuador que dentro de su Plan de Desarrollo señala como una de sus metas «Aumentar el número de personas con discapacidad y/o sustitutos insertados en el sistema laboral a 2021» [1] y es así que apoyado en la Constitución vigente, Código de Trabajo y Ley Orgánica de Discapacidades se ha logrado una integración que contribuye a la inclusión de personas con discapacidad en la sociedad. En el ámbito laboral se han creado paulatinamente mecanismos de selección en dónde se han adecuado los procesos permitiendo así la participación de personas con discapacidad procurando la equidad de género y la diversidad de discapacidad [2].

Los datos de recursos humanos proporcionan una fuente valiosa de información para el descubrimiento de conocimiento y el desarrollo de sistemas de ayuda a la toma de decisiones al momento de reclutar al personal. Hoy en día, las organizaciones tienen que luchar eficazmente en términos de costo, calidad, servicio e innovación.

El éxito de estas tareas depende de disponer de suficientes personas adecuadas con las habilidades adecuadas, desplegadas en los lugares apropiados en el momento adecuado, lo que se conoce como gestión del talento humano. Gestionar el talento de una organización se ha convertido en un desafío para los profesionales de recursos humanos, esta tarea implica muchas decisiones administrativas para elegir a la persona correcta para el trabajo correcto en el momento correcto.

A veces, este tipo de decisiones son muy inciertas y difíciles; y depende de varios factores, como la experiencia humana, el conocimiento, la preferencia y el juicio [3]. El talento se considera la capacidad de cualquier individuo para marcar una diferencia significativa en el desempeño actual y futuro de la organización [4].

El reclutamiento se dificulta cuando se analiza el proceso de inclusión laboral a personas con discapacidad. Según la Organización Mundial de la Salud (OMS) actualmente en los países en desarrollo entre el $90 \%$ y $80 \%$ de las personas con discapacidad en edad para trabajar están desempleadas, en los países industrializados la estimación es entre el $50 \%$ y $70 \%$.

Las barreras para la entrada al mercado laboral de las personas con discapacidad varían según el tipo de discapacidad, por lo que, en el proceso de inserción laboral de una persona con discapacidad, se requiere tomar medidas que garanticen el acceso y la permanencia de esta persona en su lugar de trabajo, respetando su individualidad y su tipo de discapacidad [5]. Cuando un candidato cumpla con el perfil adecuado para un cargo, la empresa debería gestionar y realizar los cambios necesarios al lugar de trabajo, ayudando así a desarrollar las capacidades de esta persona.

En el Consejo Nacional para la Igualdad de Discapacidades del Ecuador se encuentran registradas [6] a junio del 2020, 481392 personas con discapacidad de las cuales el $13 \%$ se encuentran laboralmente activas sin considerar sustitutos, de estas el 56,20\% posee discapacidad física, seguida por discapacidad auditiva $17,12 \%$, discapacidad visual un $14,31 \%$, discapacidad intelectual 8,88 \% y, finalmente, discapacidad psicosocial con un $3,50 \%$. El sector privado tiene en su nómina a 46496 personas con discapacidad y el sector público 18333 personas. Ver Figura 1.

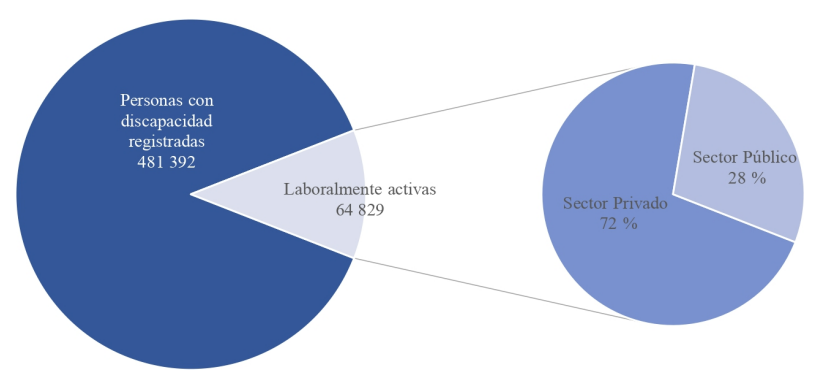

Figura 1. Personas con discapacidad registradas en el CONADIS

Según [7], el proceso de reclutamiento ha mejorado mediante la implementación de tecnologías de información, que permiten simplificar el proceso de publicar vacantes y hojas de vida, enumerar trabajos y ver posibles candidatos. Sin embargo, el proceso de reclutamiento sigue siendo imperfecto y el estudio menciona tres razones:

1. Algunos pasos del proceso de reclutamiento no están automatizados. Como resultado, los reclutadores tienen que procesar una gran cantidad (a veces cientos, o incluso miles) de hojas de vida manualmente para elegir el mejor empleado potencial.

2. Los reclutadores generalmente no toman en consideración todas las alternativas posibles para el empleado.

3. Los reclutadores se guían solo por su opinión subjetiva, por lo que no hay garantía de que el candidato elegido sea realmente la mejor opción posible para el empleador.

La contratación y selección de personal afecta directamente la calidad de los empleados. Se han realizado varios estudios sobre currículums, entrevistas, centros de evaluación, pruebas de conocimiento del trabajo, muestra de trabajo, pruebas, pruebas cognitivas y pruebas de personalidad en la gestión de recursos humanos 
para ayudar a las organizaciones a tomar mejores decisiones de selección de personal. De hecho, los enfoques de selección existentes se centran en las funciones del trabajo y el análisis de tales funciones que se definen a través de actividades y tareas específicas basadas en sus propiedades estáticas [8]. Los procesos mencionados generan una cantidad importante de datos referentes al reclutamiento, el presente estudio pretende aprovechar las técnicas de aprendizaje automático sobre estas bases de datos.

El aprendizaje automático consiste en la aplicación de técnicas de origen estadístico sobre conjuntos de datos para el aprendizaje de patrones ocultos, proyecciones o predicciones de las observaciones, mediante la aplicación de algoritmos se puede extraer información valiosa de los datos de un dominio específico de manera automática [9].

El presente trabajo está organizado de la siguiente manera, en la sección 1.1 se presentan diferentes trabajos relacionados con el área de la inteligencia artificial y su aplicación en la gestión de recursos humanos, cuando se considera oportuno se introducen diferentes conceptos. La sección 2 presenta la metodología de trabajo, detallando las técnicas que se aplicaron. La sección 3 muestra los resultados relevantes que se obtuvieron y, finalmente, la sección 4 expone las conclusiones del presente trabajo.

\subsection{Trabajos relacionados}

Las organizaciones están comenzando a adoptar y capitalizar la funcionalidad de la inteligencia artificial en sus procesos de reclutamiento [10]. Las aplicaciones de sistemas expertos o sistemas de ayuda a la toma de decisiones para seleccionar personal y el reclutamiento está incrementando [8,9,11-14].

Para la solicitud y selección de trabajo, la inteligencia artificial puede utilizar características conductuales y fisiológicas (por ejemplo, biometría) como parte del proceso general de toma de decisiones [10]. Algunos ejemplos de implementaciones son el uso de herramientas multimedia [15], sistemas de seguimiento de candidatos en línea [16], sistemas de aprendizaje automático [17], sistemas de soporte a la toma de decisiones que ayudan en el proceso completo de categorizar e identificar discapacidades [9], sin embargo, en la actualidad no se está aprovechando la riqueza de la minería de datos.

Hoy en día, existen un sinnúmero de técnicas de inteligencia artificial como la minería de datos, la analítica de datos y el descubrimiento de información en base de datos que, mediante técnicas de aprendizaje automático, proporcionan a las organizaciones tareas de predicción y clasificación, para dar soporte a la toma de decisiones, entre ellas las de gestión del talento humano.
La minería de datos hace referencia a la extracción de patrones o reglas útiles de una extensa fuente de datos, a través de la exploración automática o semiautomática y del análisis de datos [18]. Para este trabajo la aplicación de técnicas de aprendizaje automático constituye el proceso de minería de datos, según la literatura existe el consenso de clasificar las técnicas de aprendizaje automático en técnicas de aprendizaje supervisado, no supervisado y aprendizaje por refuerzo [19].

El presente trabajo se enfoca en la tarea de aplicar técnicas de aprendizaje supervisado como la clasificación y técnicas de aprendizaje no supervisado como el clustering a la base de datos recopilada en el proyecto de inclusión educativa y laboral de personas con discapacidad de la Universidad Politécnica Salesiana.

Las técnicas supervisadas permiten clasificar a un candidato con discapacidad para un puesto de trabajo como apto o no. Desde el punto de vista computacional se trata de una tarea de clasificación, sin embargo, la condición de discapacidad sesga los datos a una población muy reducida, en primer lugar, no existen suficientes datos sobre los mismos y la muestra está limitada al dominio de la ciudad de Cuenca-Ecuador.

Clasificar es una tarea de aprendizaje supervisado, donde la clase o el objetivo de clasificación es conocido. Existen varias técnicas usadas para clasificación en minería de datos como árboles de decisión, técnicas bayesianas, lógica difusa, máquinas de soporte vectorial, redes neuronales, algoritmos genéticos y el algoritmo de los vecinos más cercanos. En el presente estudio se aplican varias técnicas para determinar la que mejor se adapta a la información recolectada sobre los candidatos con discapacidad.

El clustering consiste en encontrar patrones ocultos en los datos, agrupamientos naturales que no son perceptibles en el entorno de alta dimensionalidad que presentan los conjuntos de datos modernos [19], en este caso setenta características. Debido a la alta dimensionalidad, el trabajo busca determinar correlaciones entre las distintas características para de esta manera eliminar aquellas que presenten un alto índice de correlación. Asimismo se probó con técnicas de reducción de dimensionalidad.

Es importante mencionar una línea de investigación con mucha perspectiva de crecimiento en el campo del aprendizaje automático para el apoyo a la gestión de recursos humanos, se trata del procesamiento de lenguaje natural, y como tecnología disruptiva actualmente los chatbots se ocupan de tareas como entrevistas a candidatos, capacitación a personal, atención de clientes y cualquier tarea que requiera comunicación entre personas y un agente inteligente [20]. En el área de reclutamiento de personal es importante mencionar que los chatbots son capaces de manejar una cantidad significativa de información que muchas de las veces los entrevistadores pasan por alto [21]. 


\section{Materiales y métodos}

Se utilizó como línea de base el trabajo [22], que consiste en 120 muestras de datos, con 70 características referentes a edad, sexo, nivel de educación, experiencia laboral, dominio de lengua extranjera, tipo de discapacidad y las destrezas transversales de cada candidato. El estudio original utiliza un sistema basado en reglas validado por expertos. Nuestra propuesta incorpora como novedad el aprendizaje a partir del conjunto de datos original para predecir si un candidato es apto o no para un puesto, de esta forma, el sistema mediante técnicas de aprendizaje supervisado no requiere de la validación de expertos.

La dispersión de los datos es la característica más relevante del conjunto de datos, es decir, contamos con una matriz a la cual le falta información, y dicha información no puede ser considerada como 0 en el proceso de análisis puesto que esto podría sesgar los resultados del análisis. La Tabla 1 muestra un resumen de las diferentes características del conjunto de datos.

Tabla 1. Características del conjunto de datos

\begin{tabular}{|c|c|}
\hline Tipo de información & Descripción \\
\hline $\begin{array}{l}\text { Información } \\
\text { descriptiva }\end{array}$ & $\begin{array}{c}\text { Detalles descriptivos de cada aspirante, } \\
\text { la característica más relevante en este caso } \\
\text { es la edad y el tipo de discapacidad }\end{array}$ \\
\hline $\begin{array}{l}\text { Información de } \\
\text { adaptaciones }\end{array}$ & $\begin{array}{l}\text { Hace referencia al uso de dispositivos } \\
\text { auxiliares por parte del aspirante, } \\
\text { así como su necesidad de apoyarse } \\
\text { en un intérprete o haber desarrollado } \\
\text { destrezas como el lenguaje de señas }\end{array}$ \\
\hline Estudios & Nivel de educación del aspirante \\
\hline $\begin{array}{l}\text { Información } \\
\text { del cargo }\end{array}$ & $\begin{array}{l}\text { Varias características que informan } \\
\text { sobre el detalle del cargo }\end{array}$ \\
\hline $\begin{array}{l}\text { Información de } \\
\text { la experiencia }\end{array}$ & $\begin{array}{c}\text { Características que recopilan la experiencia } \\
\text { laboral pasada del aspirante }\end{array}$ \\
\hline $\begin{array}{c}\text { Parámetros óptimos } \\
\text { para aplicar a un cargo }\end{array}$ & $\begin{array}{c}\text { Parámetros deseables para la aplicación } \\
\text { a un cargo }\end{array}$ \\
\hline $\begin{array}{l}\text { Parámetros obtenidos } \\
\text { por el aspirante }\end{array}$ & $\begin{array}{l}\text { Valoración de cada aspirante en los } \\
\text { diferentes parámetros }\end{array}$ \\
\hline $\begin{array}{l}\text { Manejo de otros } \\
\text { idiomas }\end{array}$ & Detalle sobre el dominio de otros lenguajes \\
\hline Capacitación & $\begin{array}{c}\text { Características que recopilan información } \\
\text { de las capacitaciones recibidas }\end{array}$ \\
\hline
\end{tabular}

Para el presente trabajo se imputaron los datos y se eliminaron aquellas características que no aportaban suficiente información al sistema, ya sea porque los datos recopilados representaban una cantidad insignificante de la población de estudio o porque la característica presentaba descripciones tipo texto que no aportan información al sistema. Luego de este proceso se cuenta con una matriz completa.

Para validar la eliminación de características se realizó un análisis de similaridades entre el conjunto de datos original, el que contiene los datos dispersos; y el conjunto de datos posterior a la imputación. Se utilizaron diferentes métricas de similaridad según el estudio [23], siendo relevantes para este estudio la similaridad de Pearson y la de Jaccard para el conjunto de datos completo (con características dispersas) y con el conjunto de datos modificado se consideró únicamente la similaridad de Pearson.

En la Ecuación (1) se puede apreciar cómo se calcula la similaridad entre el aspirante $u$ y el aspirante $v$ en una matriz dispersa, donde $i$ representa la enésima característica y $r$ el valor de la característica. Sea, además, $I^{\prime}$ el conjunto de todas las características en común entre $u$ y $v$.

$$
S_{P C}(u, v)=\frac{\sum_{i \in I^{\prime}}\left(r_{u i}-\bar{r}_{u}\right)\left(r_{v i}-\bar{r}_{v}\right)}{\sqrt{\sum_{i \in I^{\prime}}}\left(r_{u i}-\bar{r}_{u}\right)^{2} \sqrt{\sum_{i \in I^{\prime}}}\left(r_{v i}-\bar{r}_{v}\right)^{2}}
$$

La Ecuación (2) es utilizada para calcular la similaridad de Jaccard, en este caso lo importante es determinar si existe intersección entre las características de $u$ y $v$ sin importar la diferencia de magnitud entre sus valores.

$$
S_{J a c}(u, v)=\left|\frac{I_{u} \cap I_{v}}{I_{u} \cup I_{v}}\right|
$$

Los resultados muestran que el promedio de similaridad entre usuarios de un mismo grupo de aspirantes con la misma discapacidad está alrededor de un 0,1 tanto para la similaridad de Pearson y ligeramente inferior para la de Jaccard.

Con el conjunto de datos modificado no tiene sentido aplicar similaridad de Jaccard puesto que siempre dará uno, ya que se trata de una matriz completa, sin embargo, con la similaridad de Pearson se obtiene una mejoría en el promedio de similaridad entre usuarios de un mismo grupo de aspirantes con la misma discapacidad de 0,25 , lo cual es evidencia que la eliminación de algunas características simplificó el sistema y mejoró los resultados.

Con el nuevo conjunto de datos se procedió a generar un mapa de calor para determinar correlaciones entre las características de los aspirantes. La Figura 2 presenta el mapa de calor entre las características más relevantes, donde la intersección de cada fila y columna representa el coeficiente de correlación en el rango de $\left[\begin{array}{ll}-1 & 1\end{array}\right]$, donde valores cercanos a -1 significan una correlación fuerte inversamente proporcional y valores cercanos a 1 significan una correlación fuerte directamente proporcional, valores cercanos a 0 significan que no existe correlación. 


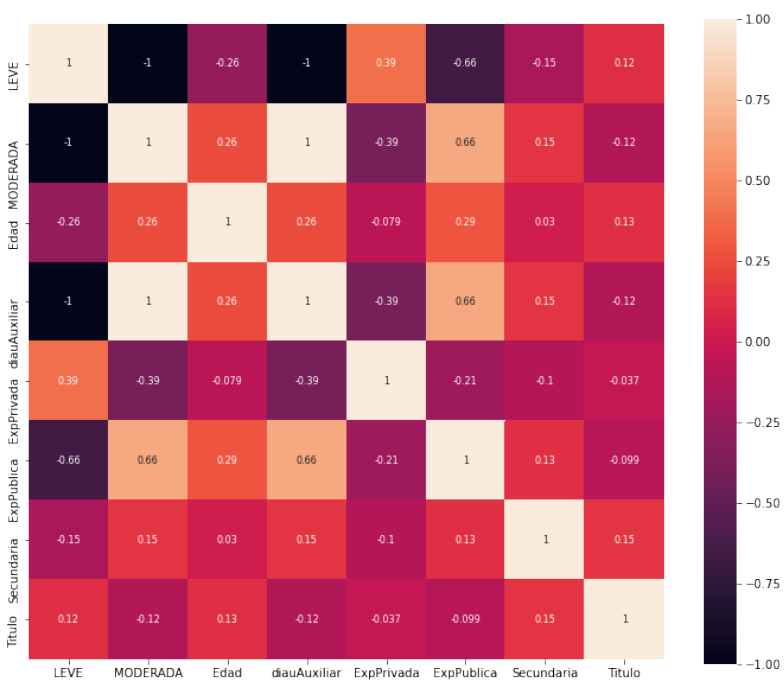

Figura 2. Mapa de calor de las correlaciones entre las características más relevantes del conjunto de datos

En este primer análisis resulta notorio la relación directa que existe entre la experiencia y el nivel de discapacidad del aspirante (moderada, leve). Por un lado, la empresa privada tiene una relación directa con el nivel de discapacidad leve, y la empresa pública tiene una relación directa con el nivel de discapacidad moderado.

Adicionalmente, se procedió a visualizar si existen relaciones entre las diferentes características mediante un gráfico de dispersión. La Figura 3 permite apreciar la dispersión de puntos entre las diferentes parejas de características del conjunto datos, la diagonal muestra el histograma de cada característica.

Luego del análisis estadístico se procedió a la analítica de los datos, donde este trabajo tiene dos objetivos, el primero es generar un modelo de clasificación para la correcta asignación de una persona a un cargo. Segundo, mediante técnicas de clustering se pretende descubrir la cohesión de los grupos de aspirantes.

En el primer caso se compararán técnicas de clasificación como la regresión logística, máquinas de soporte vectorial y el algoritmo de los vecinos más cercanos. En el segundo caso se utilizará como algoritmo de clustering $k$-means.

\section{Resultados y discusión}

\subsection{Clasificación}

Al diseñar el modelo se utilizaron diferentes técnicas de clasificación, entre ellas, un esquema de regresión logística binaria, puesto que el objetivo es intentar predecir si una persona es adecuada para el cargo o no. La regresión logística fue probada con hipótesis de tipo lineal, cuadrática y cúbica, también se implementaron las técnicas de Support Vector Machines (SVM) con un kernel gaussiano y el algoritmo de los vecinos más cercanos (KNN) para resultados con 3 y 5 vecinos.

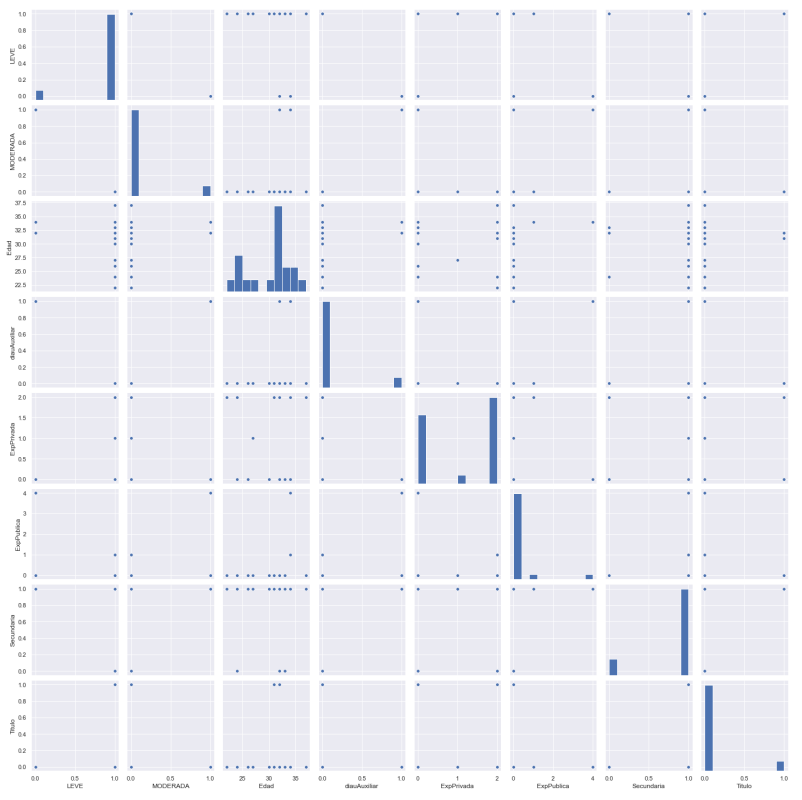

Figura 3. Gráficos de dispersión entre parejas de características

Para validar los resultados se procedió a dividir el conjunto de datos en cinco conjuntos aleatorios de train y test en una proporción de $80 \%$ y $20 \%$, respectivamente. Los resultados muestran el promedio de los cinco conjuntos aleatorios en testing. La métrica utilizada para comparar la calidad de la predicción es la precisión según la Ecuación (3), donde TP representa el número de clasificaciones realizadas correctamente (verdaderos positivos) y FP el número de clasificaciones incorrectas (falsos positivos).

$$
\text { Precisión }=\frac{T P}{T P+F P}
$$

Los resultados de los diferentes modelos se aprecian en la Tabla 2. Si bien los mejores resultados se obtienen con SVM, la diferencia en la precisión con la regresión logística no es considerable, por ello para simplificar el modelo se puede utilizar la regresión logística.

Tabla 2. Resultados de técnicas de clasificación

\begin{tabular}{ccc}
\hline Técnica & Parámetros & Precisión \\
\hline $\begin{array}{c}\text { Regresión } \\
\text { logística }\end{array}$ & Función lineal & 0,805 \\
$\begin{array}{c}\text { Regresión } \\
\text { logística }\end{array}$ & Función cuadrática & 0,815 \\
Regresión & Función cúbica & 0,818 \\
logística & Gaussian Kernel & 0,821 \\
SVM & 3 vecinos & 0,791 \\
KNN & 5 vecinos & 0,798 \\
KNN & & \\
\hline
\end{tabular}




\subsection{Clustering}

La técnica más famosa y bien conocida de hard clustering es el algoritmo k-means $[24,25]$ o hard c-means, que presenta las siguientes ventajas:

a. Conceptualmente es simple, versátil y fácil de implementar.

b. Presenta una complejidad lineal con respecto al número de elementos y clústers.

c. Se garantiza que el algoritmo termina con una tasa de convergencia cuadrática [23].

Para medir la calidad de clustering se utilizó la medida de cohesión $J_{c}$ según la Ecuación 4, donde $X$ representa la muestra del aspirante en cuestión y $C$ representa el centroide al que pertenece.

$$
J_{c}=\sum_{j=1}^{c} \sum_{t=1}^{n}\left\|X_{i}-C_{j}\right\|^{2}
$$

El algoritmo k-means utiliza el parámetro K como el indicador de los grupos a clasificar; sin embargo, el reto es determinar qué cantidad de grupos deben existir para expresar la mejor representación de los mismos. Para el conjunto de datos de los aspirantes se procedió a determinar la calidad de clustering desde $\mathrm{k}$ $=2$ hasta $\mathrm{K}=10$. En la Figura 4 mediante el método Elbow [26] se puede apreciar que no existe una clara determinación en los grupos de aspirantes; se podría considerar tanto el valor de $\mathrm{K}=3$ como el valor de $\mathrm{K}=6$ como puntos de inflexión donde la calidad del clustering se estabiliza según la curva. Esto miestra que la información que se está recopilando en el sistema requiere más trabajo, tanto en cantidad como en calidad de las características.

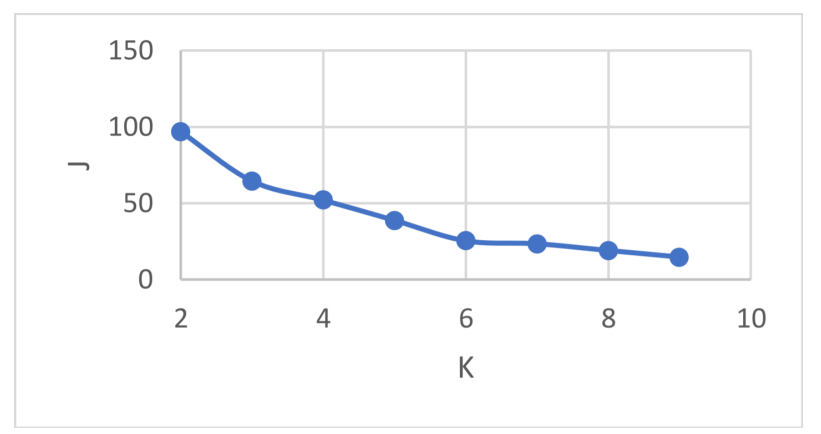

Figura 4. Elbow method para $\mathrm{k}=2$ hasta $\mathrm{k}=10$

\section{Conclusiones}

El presente estudio permite visualizar las correlaciones que existen entre diferentes variables del conjunto de datos, aportando como novedad la incorporación de técnicas de aprendizaje automático, tanto de aprendizaje supervisado para apoyar los procesos de selección del talento humano con discapacidad como de aprendizaje no supervisado para determinar en el alto espacio dimensional la cantidad de grupos de candidatos que existen en el conjunto de datos.

De acuerdo con el estudio realizado se pudo observar que el factor más determinante a la hora de conseguir empleo para una persona con discapacidad es su experiencia, es decir, haber trabajado previamente en algún lugar; en el conjunto de datos existe una correlación directa entre los aspirantes que fueron considerados aptos y la experiencia previa, sin embargo, los aspirantes que pese a ser aptos y no han sido ubicados, tienen como factor común no disponer de dicha experiencia; lo cual evidencia que para el caso de estudio, la principal característica que determina que una persona con discapacidad obtenga un cargo es esta experiencia, factor que puede ser considerado discriminatorio ya que atenta contra la igualdad de oportunidades. El conjunto de datos con el que se trabajó es considerado el más completo de su tipo para selección de personal con discapacidad, sin embargo, el presente estudio ha demostrado que se requieren características más relevantes. Es preciso mencionar que el estudio también encuentra limitaciones en la cantidad de muestras, actualmente el conjunto de datos ofrece 120 lo cual limita a las técnicas de aprendizaje automático, para el futuro sería interesante contar con un conjunto de datos mucho más grande para aplicar técnicas de aprendizaje profundo.

Varios estudios demuestran que el nivel de educación mejora la oportunidad de conseguir empleo [27], situación que no se cumple en el conjunto de datos analizado. Dichos estudios demuestran también que las personas con discapacidades tienen un nivel de educación inferior, comparado con la población general, elemento que es fácilmente verificable en este caso de estudio. La preparación académica determina que las personas con discapacidad están en desventaja para las labores que exige el mercado por razones que van más allá de la misma discapacidad. Tanto la capacitación como el nivel educativo influye notablemente en las oportunidades de trabajo, ya que en este proceso de formación se adquieren las habilidades y conocimientos dentro un área específica [28]. Los sistemas actuales continúan siendo discriminatorios para estas personas, puesto que la mayoría de organizaciones privilegia la experiencia y esto induce a una pobre valoración de las actitudes de estas personas. Situación que se ve reflejada en la calidad de los sistemas de predicción y la incapacidad para descubrir patrones de clustering claros en los datos proporcionados.

Se considera que un campo novedoso de estudio en los procesos de reclutamiento es la aplicación de chatbots en las entrevistas de selección de personal, puesto que permite eliminar elementos subjetivos que los expertos consideran que son variables de confusión 
que orientan en uno $\mathrm{u}$ otro sentido las entrevistas, factores subjetivos como la imagen personal percibida por el entrevistador, su estado de ánimo son elementos difíciles de medir y que por tanto podrían eliminarse con una adecuada selección de grupos de experimentales y de control [29].

Si bien es cierto que las políticas públicas han contribuido a la inclusión laboral de personas con discapacidad, es necesario que las empresas apoyadas en la tecnología vayan acortando las brechas que actualmente existen, asimismo eliminar en los estereotipos que impiden reconocer y potenciar las cualidades de las personas con discapacidad impidiendo así aprovechar su talento.

\section{Agradecimiento}

Los autores agradecen al grupo de investigación de inteligencia artificial y tecnologías de asistencia (GIIATA) y a Cátedra UNESCO, quienes facilitaron los datos que condujeron el presente estudio.

\section{Referencias}

[1] Consejo Nacional de Planificación, Plan Nacional de Desarrollo 2017-2021-Toda una Vida. Secretaría Nacional de Planificación y Desarrollo, Senplades. Quito - Ecuador, 2017. [Online]. Available: https://bit.ly/3isgCNb

[2] Asamblea Nacional, Ley Orgánica de Discapacidades. Registro Oficial N. ${ }^{\circ}$ 796. República del Ecuador, 2012. [Online]. Available: https://bit.ly/3cXWwch

[3] H. Jantan, A. Hamdan, and Z. Othman, "Human talent prediction in HRM using $\mathrm{C} 4.5$ classification algorithm," International Journal on Computer Science and Engineering, vol. 2, pp. 2526-2534, 2010. [Online]. Available: https://bit.ly/3aIbF1x

[4] L. Morton, "Talent management value imperatives: Strategies for execution," in Conference Board, 2005. [Online]. Available: https://bit.ly/2S4TyN0

[5] Ministerio de Relaciones Laborales, Manual de Buenas Prácticas para la inclusión laboral de personas con discapacidad. Dirección de Atención a Grupos Prioritarios. Consejo Nacional de Discapacidades. Ecuador, 2013. [Online]. Available: https://bit.ly/32S8PD4

[6] Consejo Nacional para la Igualdad de Discapacidades. (2020) Estadísticas de discapacidad. [Online]. Available: https://bit.ly/3vn30JT
[7] E. Kalugina and S. Shvydun, "An effective personnel selection model," Procedia Computer Science, vol. 31, pp. 1102-1106, 2014, 2nd International Conference on Information Technology and Quantitative Management, ITQM 2014. [Online]. Available: https://doi.org/10.1016/j.procs.2014.05.365

[8] C.-F. Chien and L.-F. Chen, "Data mining to improve personnel selection and enhance human capital: A case study in high-technology industry," Expert Systems with Applications, vol. 34, no. 1, pp. 280-290, 2008. [Online]. Available: https://doi.org/10.1016/j.eswa.2006.09.003

[9] G. Harih and N. Vujica-Herzog, "Towards an expert system for assigning optimal workplaces to workers with disabilities," in Advances in Social and Occupational Ergonomics, R. H. Goossens and A. Murata, Eds. Cham: Springer International Publishing, 2020, pp. 57-66. [Online]. Available: https://doi.org/10.1007/978-3-030-20145-6_6

[10] P. van Esch, J. S. Black, and J. Ferolie, "Marketing AI recruitment: The next phase in job application and selection," Computers in Human Behavior, vol. 90, pp. 215-222, 2019. [Online]. Available: https://doi.org/10.1016/j.chb.2018.09.009

[11] R. Storey Hooper, T. P. Galvin, R. A. Kilmer, and J. Liebowitz, "Use of an expert system in a personnel selection process," Expert Systems with Applications, vol. 14, no. 4, pp. 425-432, 1998. [Online]. Available: https://doi.org/10.1016/S0957-4174(98)00002-5

[12] M. Nussbaum, M. Singer, R. Rosas, M. Castillo, E. Flies, R. Lara, and R. Sommers, "Decision support system for conflict diagnosis in personnel selection," Information \& Management, vol. 36, no. 1, pp. 55-62, 1999. [Online]. Available: https://doi.org/10.1016/S0378-7206(99)00007-5

[13] S. M. C. Loureiro, J. Guerreiro, and I. Tussyadiah, "Artificial intelligence in business: State of the art and future research agenda," Journal of Business Research, vol. 129, pp. 911-926, 2021. [Online]. Available: https://doi.org/10.1016/j.jbusres.2020.11.001

[14] H. J. Wilson and P. R. Daugherty, "Collaborative intelligence: Humans and AI are joining forces," Harvard Business Review, 2018. [Online]. Available: https://bit.ly/3npoJOx

[15] S. T. Hunter, N. D. Shortland, M. P. Crayne, and G. S. Ligon, "Recruitment and selection in violent extremist organizations: Exploring what industrial and organizational psychology might contribute." The American psychologist, vol. 72, pp. 242-254, Apr 2017. [Online]. Available: https://doi.org/10.1037/amp0000089 
[16] A. Eckhardt, S. Laumer, C. Maier, and T. Weitzel, "The transformation of people, processes, and it in e-recruiting," Employee Relations, vol. 36, no. 4, pp. 415-431, Apr. 2021. [Online]. Available: https://doi.org/10.1108/ER-07-2013-0079

[17] N. Herbst, S. Becker, S. Kounev, H. Koziolek, M. Maggio, A. Milenkoski, and E. Smirni, Metrics and Benchmarks for Self-aware Computing Systems. Cham: Springer International Publishing, 2017, pp. 437-464. [Online]. Available: https://doi.org/10.1007/978-3-319-47474-8_14

[18] M. J. A. Berry and G. S. Linoff, Data Mining Techniques: For Marketing, Sales, and Customer Relationship Management. John Wiley \& Sons, 2004. [Online]. Available: https://bit.ly/3tXJh37

[19] P.-N. Tan, M. Steinbach, and V. Kumar, Introduction to data mining. Pearson Education, 2013. [Online]. Available: https://bit.ly/3voKVeG

[20] J. Hill, W. Randolph Ford, and I. G. Farreras, "Real conversations with artificial intelligence: A comparison between human-human online conversations and human-chatbot conversations," Computers in Human Behavior, vol. 49, pp. 245-250, 2015. [Online]. Available: https://doi.org/10.1016/j.chb.2015.02.026

[21] A. M. Rahman, A. A. Mamun, and A. Islam, "Programming challenges of chatbot: Current and future prospective," in 2017 IEEE Region 10 Humanitarian Technology Conference (R10-HTC), 2017, pp. 75-78. [Online]. Available: https://doi.org/10.1109/R10-HTC.2017.8288910

[22] M. Rodas-Tobar, C. Bernal-Bravo, M. AndrésRomero, A. Pinos-Figueroa, P. Vidal-Mogrovejo, A. León-Pesántez, V. Robles-Bykbaev, and F. Pesántez-Avilés, "An expert system to support the provisioning of staff with disabilities in industry," in 2018 IEEE Biennial Congress of Argentina (ARGENCON), 2018, pp. 1-6. [Online]. Available: https: //doi.org/10.1109/ARGENCON.2018.8646043
[23] R. X. Bojorque Chasi, "Clustering de sistemas de recomendación mediante técnicas de factorization matricial," Ph.D. dissertation, 2020. [Online]. Available: https: //doi.org/10.20868/UPM.thesis.58156

[24] J. MacQueen, "Some methods for classification and analysis of multivariate observations," in Proceedings of the fifth Berkeley symposium on mathematical statistics and probability, 1967. [Online]. Available: https://bit.ly/3xrH16A

[25] S. Zahra, M. A. Ghazanfar, A. Khalid, M. A. Azam, U. Naeem, and A. PrugelBennett, "Novel centroid selection approaches for kmeans-clustering based recommender systems," Information Sciences, vol. 320, pp. 156-189, 2015. [Online]. Available: https://doi.org/10.1016/j.ins.2015.03.062

[26] M. A. Syakur, B. K. Khotimah, E. M. S. Rochman, and B. D. Satoto, "Integration k-means clustering method and elbow method for identification of the best customer profile cluster," in IOP Conference Series: Materials Science and Engineering, vol. 336, 2017. [Online]. Available: https: //doi.org/10.1088/1757-899x/336/1/012017

[27] T. Boman, A. Kjellberg, B. Danermark, and E. Boman, "Employment opportunities for persons with different types of disability," Alter, vol. 9, no. 2, pp. 116-129, 2015. [Online]. Available: https://doi.org/10.1016/j.alter.2014.11.003

[28] M. A. Espinoza Mina and D. Gallegos Barzola, "Inserción laboral de las personas con discapacidad en Ecuador," Espacios, vol. 39, no. 51, 2018. [Online]. Available: https://bit.ly/3sXKMwS

[29] M. Reynolds, "Ai coach helps chatbots seem more human," New Scientist, vol. 235, no. 3135, p. 14, 2017. [Online]. Available: https://doi.org/10.1016/S0262-4079(17)31407-0 